\title{
Acute hypoxemic respiratory failure in immunocompromised patients : the Efraim multinational prospective cohort study
}

\section{Azoulay, Elie}

2017-12

Azoulay , E, Pickkers , P , Soares , M , Perner , A, Rello , J , Bauer , P R, van de Louw , A , Hemelaar, P , Lemiale , V , Taccone , F S , Loeches , I M , Meyhoff , T S , Salluh , J , Schellongowski , P, Rusinova , K, Terzi , N , Mehta , S, Antonelli , M , Kouatchet , A, Barratt-Due, A, Valkonen, M , Landburg, P P, Bruneel , F , Bukan, R B , Pene , F , Metaxa , V , Moreau , A S , Souppart , V , Burghi , G , Girault , C , Silva , U V A, Montini , L , Barbier , F, Nielsen , L B , Gaborit, B, Mokart, D, Chevret, S, Efraim Investigators \& Nine-I Study Grp 2017 , ' Acute hypoxemic respiratory failure in immunocompromised patients : the Efraim multinational prospective cohort study ' , Intensive Care Medicine, vol. 43 , no. 12 , pp. 1808-1819 . https://doi.org/10.1007/s00134-017-4947-1

http://hdl.handle.net/10138/283172

https://doi.org/10.1007/s00134-017-4947-1

publishedVersion

Downloaded from Helda, University of Helsinki institutional repository.

This is an electronic reprint of the original article.

This reprint may differ from the original in pagination and typographic detail.

Please cite the original version. 


\title{
SEVEN-DAY PROFILE PUBLICATION
}

\section{Acute hypoxemic respiratory failure in immunocompromised patients: the Efraim multinational prospective cohort study}

Elie Azoulay ${ }^{1 *} \mathbb{D}$, Peter Pickkers ${ }^{2}$, Marcio Soares ${ }^{3}$, Anders Perner ${ }^{4}$, Jordi Rello ${ }^{5}$, Philippe R. Bauer ${ }^{6}$, Andry van de Louw ${ }^{7}$, Pleun Hemelaar ${ }^{2}$, Virginie Lemiale ${ }^{1}$, Fabio Silvio Taccone ${ }^{8}$, Ignacio Martin Loeches ${ }^{9,10}$, Tine Sylvest Meyhoff ${ }^{4}$, Jorge Salluh ${ }^{3}$, Peter Schellongowski ${ }^{11}$, Katerina Rusinova ${ }^{12}$, Nicolas Terzi ${ }^{13}$, Sangeeta Mehta ${ }^{14}$, Massimo Antonelli ${ }^{15}$, Achille Kouatchet ${ }^{16}$, Andreas Barratt-Due ${ }^{17}$, Miia Valkonen ${ }^{18}$, Precious Pearl Landburg ${ }^{19}$, Fabrice Brunee ${ }^{20}$, Ramin Brandt Bukan ${ }^{21}$, Frédéric Pène ${ }^{22}$, Victoria Metaxa ${ }^{23}$, Anne Sophie Moreau ${ }^{24}$, Virginie Souppart ${ }^{1}$, Gaston Burghi ${ }^{25}$, Christophe Girault ${ }^{26}$, Ulysses V. A. Silva ${ }^{27}$, Luca Montini ${ }^{15}$, François Barbier ${ }^{28}$, Lene B. Nielsen ${ }^{29,30}$, Benjamin Gaborit ${ }^{31}$, Djamel Mokart ${ }^{32}$ and Sylvie Chevret ${ }^{33}$ for the Efraim investigators and the Nine-I study group

@ 2017 Springer-Verlag GmbH Germany and ESICM

\begin{abstract}
Background: In immunocompromised patients with acute hypoxemic respiratory failure (ARF), initial management aims primarily to avoid invasive mechanical ventilation (IMV).

Methods: To assess the impact of initial management on IMV and mortality rates, we performed a multinational observational prospective cohort study in 16 countries (68 centers).

Results: A total of 1611 patients were enrolled (hematological malignancies 51.9\%, solid tumors $35.2 \%$, systemic diseases 17.3\%, and solid organ transplantation 8.8\%). The main ARF etiologies were bacterial (29.5\%), viral (15.4\%), and fungal infections (14.7\%), or undetermined (13.2\%). On admission, 915 (56.8\%) patients were not intubated. They received standard oxygen $(N=496,53.9 \%$ ), high-flow oxygen (HFNC, $N=187,20.3 \%$ ), noninvasive ventilation (NIV, $N=153,17.2 \%)$, and NIV + HFNC $(N=79,8.6 \%)$. Factors associated with IMV included age (hazard ratio $=0.92 /$ year, 95\% Cl 0.86-0.99), day-1 SOFA (1.09/point, 1.06-1.13), day-1 $\mathrm{PaO}_{2} / \mathrm{FiO}_{2}(1.47,1.05-2.07)$, ARF etiology (Pneumocystis jirovecii pneumonia $(2.11,1.42-3.14)$, invasive pulmonary aspergillosis $(1.85,1.21-2.85)$, and undetermined cause (1.46, 1.09-1.98). After propensity score matching, HFNC, but not NIV, had an effect on IMV rate (HR $=0.77,95 \% \mathrm{Cl} 0.59$ $1.00, p=0.05)$. ICU, hospital, and day-90 mortality rates were $32.4,44.1$, and $56.4 \%$, respectively. Factors independently
\end{abstract}

\footnotetext{
*Correspondence: elie.azoulay@sls.aphp.fr

${ }^{1}$ Medical Intensive Care Unit, APHP, Hôpital Saint-Louis, Famirea Study Group, ECSTRA team, and Clinical Epidemiology, UMR 1153, Center of Epidemiology and Biostatistics, Sorbonne Paris Cité, CRESS, INSERM, Paris Diderot Sorbonne University, Paris, France

Full author information is available at the end of the article

The members of the Efraim investigators and the Nine-l study group are listed in the Acknowledgements and in ESM 2.

All authors substantially contributed to the design of the study, acquisition, analysis, and interpretation of data, drafted and revised the manuscript for important intellectual content, approved the final version, and agreed to be accountable for all aspects of the work.
}

\section{县 Springer}


associated with hospital mortality included age (odds ratio $=1.18 /$ year, 1.09-1.27), direct admission to the ICU (0.69, 0.54-0.87), day-1 SOFA excluding respiratory score (1.12/point, 1.08-1.16), $\mathrm{PaO}_{2} / \mathrm{FiO}_{2}<100$ (1.60, 1.03-2.48), and undetermined ARF etiology (1.43, 1.04-1.97). Initial oxygenation strategy did not affect mortality; however, IMV was associated with mortality, the odds ratio depending on IMV conditions: NIV + HFNC failure (2.31, 1.09-4.91), first-line IMV $(2.55,1.94-3.29)$, NIV failure $(3.65,2.05-6.53)$, standard oxygen failure $(4.16,2.91-5.93)$, and HFNC failure (5.54, 3.27-9.38).

Conclusion: HFNC has an effect on intubation but not on mortality rates. Failure to identify ARF etiology is associated with higher rates of both intubation and mortality. This suggests that in addition to selecting the appropriate oxygenation device, clinicians should strive to identify the etiology of ARF.

Keywords: Noninvasive ventilation, High flow oxygen, Hematological malignancies, Transplantation, Systemic diseases, Pneumocystis

\section{Introduction}

The number of living immunosuppressed adults is increasing [1]. This is the result of new indications and development of immunosuppressive treatments in autoimmune conditions and solid organ transplantation [2, $3]$, of greater life expectancy in patients with malignancies $[4,5]$ and the high number of averted deaths from cancer over the last decade [6]. Immunosuppression, either related to the underlying condition or to its treatments, increases the risk and severity of infections, primary bacterial, viral, or opportunistic infections, as well as reactivation of latent infections [7, 8]. Moreover, noninfectious causes of critical illness may also be triggered by specific infiltration from the underlying disease or by drug-related organ toxicity.

Acute hypoxemic respiratory failure (ARF) is the leading cause of ICU admission in immunocompromised patients $[4,9,10]$. It is also one of the most challenging conditions as initial management must optimize oxygenation, identify the ARF etiology, and support associated organ dysfunction at the same time [4]. Over the last decade, small and single-center studies, or post hoc analyses in limited numbers of patients provided conflicting information on what can be expected from high-flow oxygen administered through a nasal cannula (HFNC) in immunocompromised patients with ARF [11-13]. Moreover, a randomized clinical trial suggested no benefit from noninvasive ventilation (NIV) in immunocompromised patients with ARF [14]. Furthermore, NIV use alone or in association with HFNC was associated with a dramatic increase in both the need for invasive mechanical ventilation (IMV) and in hospital mortality, raising concerns about the selection of initial oxygenation strategy in these patients [15].

Mortality remains remarkably high, reaching 60\% if ICU admission is delayed [16], in case of NIV failure, invasive fungal infection, or when the ARF etiology remains undetermined [17-22]. Thus, clinicians are left with a difficult dilemma of either preventing intubation by all means, or giving priority to identifying ARF etiology, possibly including intubation for diagnostic procedures such as fiberoptic bronchoscopy and bronchoalveolar lavage (FO-BAL). Although bronchoscopy and noninvasive diagnostic tests perform equally [23], FO-BAL may be needed in several cases [18].

To guide clinical practice and inform the clinician on whether initial management should give priority to identifying ARF etiology or to avoiding IMV, we performed a large prospective multinational cohort study. Immunocompromised patients with hypoxemic ARF were recruited from 68 ICUs in 16 countries. We hypothesized that oxygenation strategy influences outcomes as much as recognizing the etiology of ARF. Need for IMV and hospital mortality were our primary endpoints.

\section{Patients and methods \\ Main objective}

The primary objective of this study was to assess whether initial management (i.e., oxygenation and diagnostic strategies) affects the proportion of patients requiring intubation and mechanical ventilation or hospital mortality.

\section{Study design and participants}

Efraim was a multinational, observational prospective cohort study performed by the Nine-I (Caring for critically ill immunocompromised patients) study group. This group includes critical care physicians from 16 countries who have extensive experience in the management of various groups of critically ill immunocompromised patients. Physician participation was voluntary, without financial incentive. Participating providers obtained institutional review board (IRB) approval from their institutions in accordance with local ethics regulations.

Inclusion criteria were 18 years or older; acute hypoxemic respiratory failure $\left(\mathrm{PaO}_{2}<60 \mathrm{mmHg}\right.$ or $\mathrm{SpO}_{2}<90 \%$ on room air, or tachypnea $>30 / \mathrm{min}$, or labored breathing or respiratory distress or dyspnea at rest or cyanosis); need for more than $6 \mathrm{~L} / \mathrm{min}$ oxygen; respiratory symptom duration less than $72 \mathrm{~h}$; 
and non-AIDS-related immune deficiency defined as hematologic malignancy or solid tumor (active or in remission for less than 5 years, including recipients of autologous or allogeneic stem cell transplantation), solid organ transplant, long-term (> 30 days) or high-dose (> $1 \mathrm{mg} / \mathrm{kg} /$ day) steroids, or any immunosuppressive drug for more than 30 days. Exclusion criteria included postoperative acute respiratory failure (within 6 days of surgery), admission after a cardiac arrest, ICU admission only to secure bronchoscopy, or refusal of the patient or family to participate in the study. Primary endpoints were the need for IMV in patients not intubated on ICU admission and all-cause hospital mortality.

After IRB approval, participating ICUs enrolled patients between 5 November 2015 and 1 July 2016. A standardized paper case report was prepared by investigators and tested in 35 patients. After feedback and corrections, the case report form was sent to participating ICUs and, once completed, it was sent back to the coordinating center in Paris for data entry by specialized technicians used to handling data on critically ill immunocompromised patients. The study was funded by the Groupe de Recherche en Reanimation Respiratoire Onco-Hématologique (GRRR-OH), an academic not-forprofit French organization.

\section{Study treatments}

All management decisions were independently made by the attending physicians according to standard practice in each ICU. Diagnostic tests to identify the cause of respiratory failure were invasive (FO-BAL), noninvasive (blood and sputa cultures for bacteria and fungi, serum and urine antigens, PCR in blood, serum and nasopharyngeal aspirates, high resolution CT scan, echocardiography, serology, and specific tests according to each situation), or both [14, 18-20, 23]. All diagnoses were reviewed by two study investigators for coherence and for alignment with established definitions. Oxygenation modalities and the use of NIV or HFNC were at the discretion of the primary team. Management of associated organ dysfunction and handling of immunosuppressive drugs or chemotherapy were done as per local preferences. The decision to intubate was not controlled by the study.

Patient's code status on ICU admission was recorded as full code management, time-limited trial, do not intubate (DNI), do not resuscitate (DNR), or palliative.

\section{Study outcomes}

The two primary study outcomes were the need for IMV and all-cause mortality before hospital discharge. Patients were enrolled immediately at ICU admission and the data in the tables and figures were collected prospectively using the paper case report form. The sequential organ failure assessment (SOFA) score was recorded within $24 \mathrm{~h}$ of admission [24]. We did not use the term acute respiratory distress syndrome (ARDS) as defined on the basis of the 2012 definition because most patients were not ventilated at day 1 and were not receiving positive end-expiratory pressure (PEEP) [25].

\section{Statistical analysis}

We based our computation on the potential effect size demonstrated using logistic regression modelling, with fixed sample size, and prevalence of hospital mortality ranging from 0.15 to 0.5 . It showed that a sample size of 1500 will reach an $80 \%$ power to detect an odds ratio (OR) of 1.3 with balanced groups, and OR of at least 1.5 with imbalanced groups of 1:4.

Continuous variables were described as medians [interquartile ranges (IQRs)] and categorical variables as proportions. Data management allowed checking for data inconsistencies that were solved by consensus.

Comparisons of proportions between groups were made using the $\chi^{2}$ test. Comparisons of continuous variables between groups were made using the Wilcoxon rank-sum test.

To analyze the predictive factors of intubation, the analysis has to deal with death prior to intubation that acts as a competing event for intubation. Thus, cumulative incidence curves were plotted, and compared across baseline groups using the Gray test. Predictive factors were assessed through the use of cause-specific Cox models; univariate models were fitted, and then those associated with the outcome at the $5 \%$ level were introduced into a multivariable model, with variable selection based on the Akaike criterion. The analysis of hospital mortality was based on logistic models, with model selection similar to that described above for intubation outcome. In this model, SOFA score was introduced without items of the respiratory score to assess the impact of $\mathrm{PaO}_{2} / \mathrm{FiO}_{2}$ ratio in immunocompromised patients [20]. To assess the benefit of the use on ICU admission of NIV or HFNC in non-mechanically ventilated patients, we used a propensity score approach to control for observed confounding factors that might influence both group assignment and outcome. Each model was performed separately as follows. Briefly, we estimated the propensity score of being administered NIV (HFNC, resp.) at the time of ICU admission using a logistic regression model including potential predictors as selected by prognostic analyses described above. To handle missing values in confounders, multiple imputation with chained equation was used, where propensity score for each patient was averaged across 30 completed datasets while propensity score matching used these averaged scores to estimate 


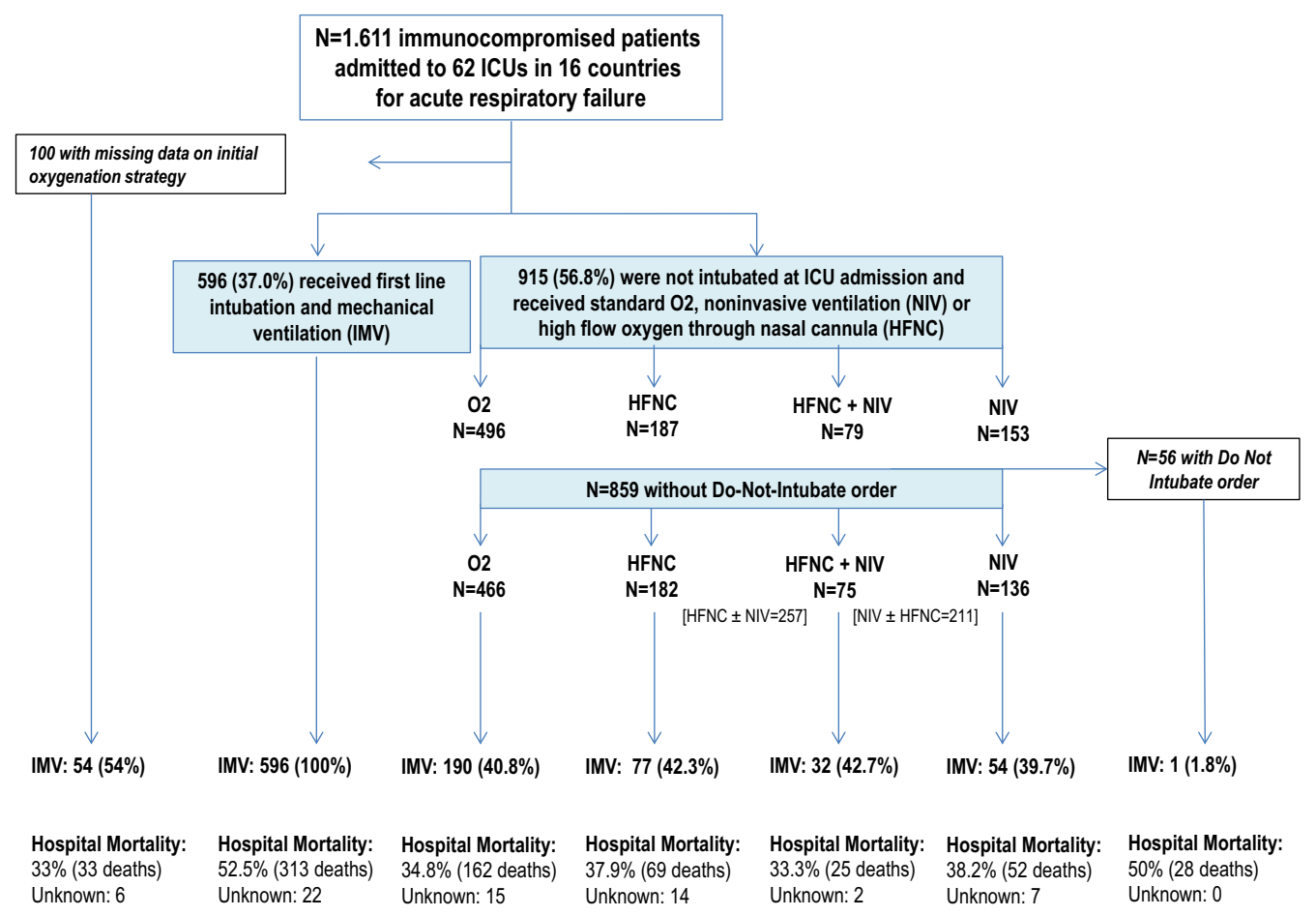

Fig. 1 Study flowchart

the treatment effect. A 1:1 matching algorithm without replacement within a caliper of 0.15 standard deviation of the logit of the propensity score was used; balances in confounders before and after matching were checked using standardized mean differences. Estimates of the effect of NIV on outcomes, accounting for paired data, were based on either a cause-specific frailty Cox model or logistic regression model by generalized estimating equations, respectively.

Primary analyses were performed on the complete cases, assuming missing completely at random covariates. Then, sensitivity analyses for such assumptions were performed, based on multiple imputation with chained equation. To incorporate potential center effects, models with centers introduced as clusters or random variables were also fitted. Last, we performed exploratory subset analyses, restricting ourselves to patients with hematological malignancies or full code status.

All reported $P$ values are 2 -sided; $P<0.05$ was considered statistically significant. All analyses were performed using R version 3.3.2 (http://www.R-project.org/).

\section{Results}

Over the 8-month study period, 1611 patients [ $60 \%$ male, median age 63 years old (IQR 54-71)] were enrolled in the 68 participating ICUs. Supplemental Fig. 1 shows the number of patients recruited per country.
Immunosuppression was related to hematological malignancy [837 (51.9\%), including 103 allogeneic and 152 autologous hematopoietic stem cell transplantations], solid tumor [567 (35.2\%)], systemic rheumatic disease [278 (17.3\%)], and solid organ transplant [142 (8.8\%)]. Fourteen $(0.9 \%)$ patients received immunosuppressive drugs for rare diseases. More than one condition was reported in 199 patients. Half the patients were newly diagnosed, $25 \%$ were in second-line therapy, $15 \%$ were in remission, and $15 \%$ had received elements of palliative care. The resuscitation status was full code in 1292 (80.2\%), time-limited trial in 69 (4.3\%), DNI in 58 (3.6\%), DNR in $56(3.5 \%)$, and the code status was not established in $136(8.4 \%)$ cases.

Most patients were admitted from the wards (57\%); however, $348(21.6 \%)$ were directly admitted from the emergency department or by ambulances. On admission, respiratory rate was $30 / \mathrm{min}(25-36), \mathrm{SpO}_{2}$ was $92 \%$ through $8 \mathrm{~L} / \mathrm{min}(4-15) \mathrm{O}_{2}$ or a $\mathrm{FiO}_{2}$ of $50 \%(40-80)$. $\mathrm{PaO}_{2} / \mathrm{FiO}_{2}$ ratio was $151(100-233)$ and SOFA score was 7 (4-10) on day 1. IMV was needed in 1004 (62.3\%) patients throughout the ICU stay. ICU, hospital, and day90 mortality rates were $32.4 \%$ (522 deaths), $44.1 \%$ (682 deaths, 66 missing information), and 56.4\% (771 deaths, 245 missing), respectively.

Figure 1 depicts oxygenation and ventilation modalities following ICU admission. First-line IMV was offered 
Table 1 Characteristics of the 915 patients who were not intubated on ICU admission

\begin{tabular}{|c|c|c|c|c|c|}
\hline Numbers (\%) or median (IQR) & $\begin{array}{l}\text { Standard oxygen } \\
\left(\mathrm{O}_{2}, \mathrm{~N}=496\right)\end{array}$ & $\begin{array}{l}\text { High-flow oxygen } \\
\text { (HFNC, } N=187 \text { ) }\end{array}$ & $\begin{array}{l}\text { Noninvasive ventilation } \\
(\mathrm{NIV}, \mathrm{N}=153)\end{array}$ & HFNC + NIV $(N=79)$ & $P$ value \\
\hline Age (years) & $63.7(54.4-70.8)$ & $62.1(52.7-69.8)$ & $65.1(56.6-71.9)$ & $64.1(52-71)$ & 0.40 \\
\hline Female gender & $184(37.1 \%)$ & $63(33.7 \%)$ & $63(41.2 \%)$ & $41(51.9 \%)$ & 0.03 \\
\hline Chronic pulmonary disease & $62(12.5 \%)$ & $23(12.3 \%)$ & $42(27.4 \%)$ & $11(13.9 \%)$ & 0.0002 \\
\hline Chronic cardiac failure & $107(21.6 \%)$ & $31(16.6 \%)$ & $41(26.8 \%)$ & $23(29.1 \%)$ & 0.09 \\
\hline Days since symptoms onset & $1(0-4)$ & $2(0-8)$ & $1(0-3)$ & $2(1-5)$ & 0.0001 \\
\hline Location before ICU admission & & & & & 0.05 \\
\hline Emergency department & $112(22.6 \%)$ & $36(19.3 \%)$ & $48(31.4 \%)$ & $9(11.4 \%)$ & \\
\hline Hospital wards & $307(61.9 \%)$ & $116(62 \%)$ & $89(58.2 \%)$ & $55(69.6 \%)$ & \\
\hline Other ICUs & $22(4.4 \%)$ & $2(1.1 \%)$ & $3(1.9 \%)$ & $1(1.3 \%)$ & \\
\hline Other locations & $55(11.1 \%)$ & $33(17.6 \%)$ & $13(8.5 \%)$ & $14(17.7 \%)$ & \\
\hline Nightly or weekend admissions & $304(61.3 \%)$ & $118(63.1 \%)$ & $82(53.6 \%)$ & $57(72.1 \%)$ & 0.06 \\
\hline \multicolumn{6}{|l|}{ Underlying condition } \\
\hline Hematological malignancy & $274(55.2 \%)$ & $110(58.8 \%)$ & $73(47.7 \%)$ & $46(58.2 \%)$ & 0.11 \\
\hline Solid tumor & $158(31.8 \%)$ & $54(28.9 \%)$ & $49(32 \%)$ & $23(29.1 \%)$ & 0.84 \\
\hline Systemic diseases & $84(16.9 \%)$ & $36(19.3 \%)$ & $32(20.9 \%)$ & $14(17.7 \%)$ & 0.84 \\
\hline Solid organ transplant & $42(8.5 \%)$ & $23(12.3 \%)$ & $20(13.1 \%)$ & $6(7.6 \%)$ & 0.21 \\
\hline Long-term/high-dose steroids & $384(77.4 \%)$ & $148(79.1 \%)$ & $114(74.5 \%)$ & $47(59.4 \%)$ & 0.13 \\
\hline Neutropenia & $78(15.7 \%)$ & $34(18.2 \%)$ & $24(15.7 \%)$ & $20(25.3 \%)$ & 0.18 \\
\hline \multicolumn{6}{|c|}{ Hematopoietic stem cell transplantation } \\
\hline Autologous & $36(7.3 \%)$ & $13(6.9 \%)$ & $7(4.6 \%)$ & $6(7.6 \%)$ & 0.13 \\
\hline Allogeneic & $36(7.3 \%)$ & $26(13.9 \%)$ & $15(9.8 \%)$ & $12(15.2 \%)$ & \\
\hline Days since diagnosis & $142(15-672)$ & $204(26-725)$ & $118(24-671)$ & $81(1-424)$ & 0.20 \\
\hline Newly diagnosed disease & $150(30.2 \%)$ & $51(27.3 \%)$ & $58(37.9 \%)$ & $20(25.3 \%)$ & 0.28 \\
\hline SOFA score on ICU admission & $5(3-8)$ & $6(4-8)$ & $5(3-8)$ & $5(4-8)$ & 0.04 \\
\hline $\mathrm{PaO}_{2} / \mathrm{FiO}_{2}$ on day 1 & $173(136-220)$ & $150(100-173)$ & $168.5(112-188)$ & $128(100-173)$ & $<0.0001$ \\
\hline \multicolumn{6}{|c|}{ Acute respiratory failure (ARF) etiology } \\
\hline Bacterial infection & $131(26.4 \%)$ & $27(14.4 \%)$ & $63(41.2 \%)$ & $21(26.7 \%)$ & 0.001 \\
\hline Viral infection & $70(14.1 \%)$ & $38(20.3 \%)$ & $30(19.6 \%)$ & $14(17.7 \%)$ & 0.18 \\
\hline Pneumocystis pneumonia & $21(4.2 \%)$ & $18(9.6 \%)$ & $6(3.9 \%)$ & $10(12.6 \%)$ & 0.005 \\
\hline Invasive pulmonary aspergillosis & $20(4.0 \%)$ & $7(3.7 \%)$ & $7(4.6 \%)$ & $3(3.8 \%)$ & 1 \\
\hline Invasive fungal infection & $65(13.1 \%)$ & $39(20.8 \%)$ & $21(13.7 \%)$ & $13(16.4 \%)$ & 0.05 \\
\hline Disease-related infiltrates & $52(10.5 \%)$ & $20(10.7 \%)$ & $12(7.8 \%)$ & $9(11.4 \%)$ & 0.71 \\
\hline Cardiogenic pulmonary edema & $44(8.9 \%)$ & $8(4.3 \%)$ & $16(10.4 \%)$ & $8(10.1 \%)$ & 0.20 \\
\hline Undetermined & $63(12.7 \%)$ & $28(15 \%)$ & $14(9.1 \%)$ & $12(15.2 \%)$ & 0.53 \\
\hline More than one ARF etiology & $71(14.3 \%)$ & $34(18.2 \%)$ & $30(19.6 \%)$ & $13(16.4 \%)$ & 0.39 \\
\hline Code status on ICU admission & & & & & 0.62 \\
\hline Full code & $442(89.1 \%)$ & $173(92.5 \%)$ & $124(81.1 \%)$ & $65(82.3 \%)$ & \\
\hline Time-limited trial & $12(2.4 \%)$ & $7(3.7 \%)$ & $3(1.9 \%)$ & $8(10.1 \%)$ & \\
\hline DNI/DNR & $42(8.5 \%)$ & $7(3.7 \%)$ & $26(17 \%)$ & $6(7.6 \%)$ & \\
\hline Need for intubation (overall) & $202(40.7 \%)$ & $77(41.2 \%)$ & $61(39.9 \%)$ & $34(43.0 \%)$ & 0.95 \\
\hline Intubation $\geq$ day 3 & $99 / 202(49 \%)$ & $45 / 77(58.4 \%)$ & $29 / 61(47.5 \%)$ & $17 / 34(50 \%)$ & 0.74 \\
\hline ICU mortality & $107(21.6 \%)$ & $55(29.4 \%)$ & $39(25.5 \%)$ & $20(25.3 \%)$ & 0.28 \\
\hline Hospital mortality (38 missing) & $162(32.7 \%)$ & $69(36.9 \%)$ & $62(40.5 \%)$ & $27(34.2 \%)$ & 0.54 \\
\hline Day-90 mortality (140 missing) & $198(39.9 \%)$ & $78(41.7 \%)$ & $74(48.4 \%)$ & $36(45.6 \%)$ & 0.85 \\
\hline
\end{tabular}

to 596 (37\%) patients who were intubated on ICU admission (Fig. 1). Among the 915 patients not intubated on ICU admission, 496 (54.2\%) received standard oxygen only, 187 (20.4\%) HFNC only, 153 (16.7\%) NIV only, and 79 (8.6\%) both HFNC + NIV. Table 1 shows patients' characteristics in these four groups. Patients receiving 
HFNC at day 1 were more severely hypoxemic than other patients $\left(\mathrm{PaO}_{2} / \mathrm{FiO}_{2}=122\right.$ (88-183) vs. 163 (103-250), $P<0.0001)$. In patients receiving continuous standard oxygen, oxygen flow was $8 \mathrm{~L} / \mathrm{min}(4-15), 5 \mathrm{~L} / \mathrm{min}(3-8)$, and $4 \mathrm{~L} / \mathrm{min}(2-7)$, at day 1 , day 2 , and day 3 , respectively. In patients receiving NIV, NIV duration was $8 \mathrm{~h} /$ day (5-14), $8 \mathrm{~h} /$ day (4-15), and $7 \mathrm{~h} /$ day (3-15), at day 1 , day 2 , and day 3 , respectively. FiO2, PEEP, and pressure support at day 1 were $60 \%(40-80), 8 \mathrm{cmH}_{2} \mathrm{O}(5-10)$, and $14 \mathrm{cmH}_{2} \mathrm{O}(9-16)$. In patients receiving continuous HFNC, oxygen flow was $50 \mathrm{~L} / \mathrm{min}(40-50)$ on day 1 and day 2 , and $40 \mathrm{~L} / \mathrm{min}(30-50)$ on day 3 . $\mathrm{HFNC}-\mathrm{FiO}_{2}$ was
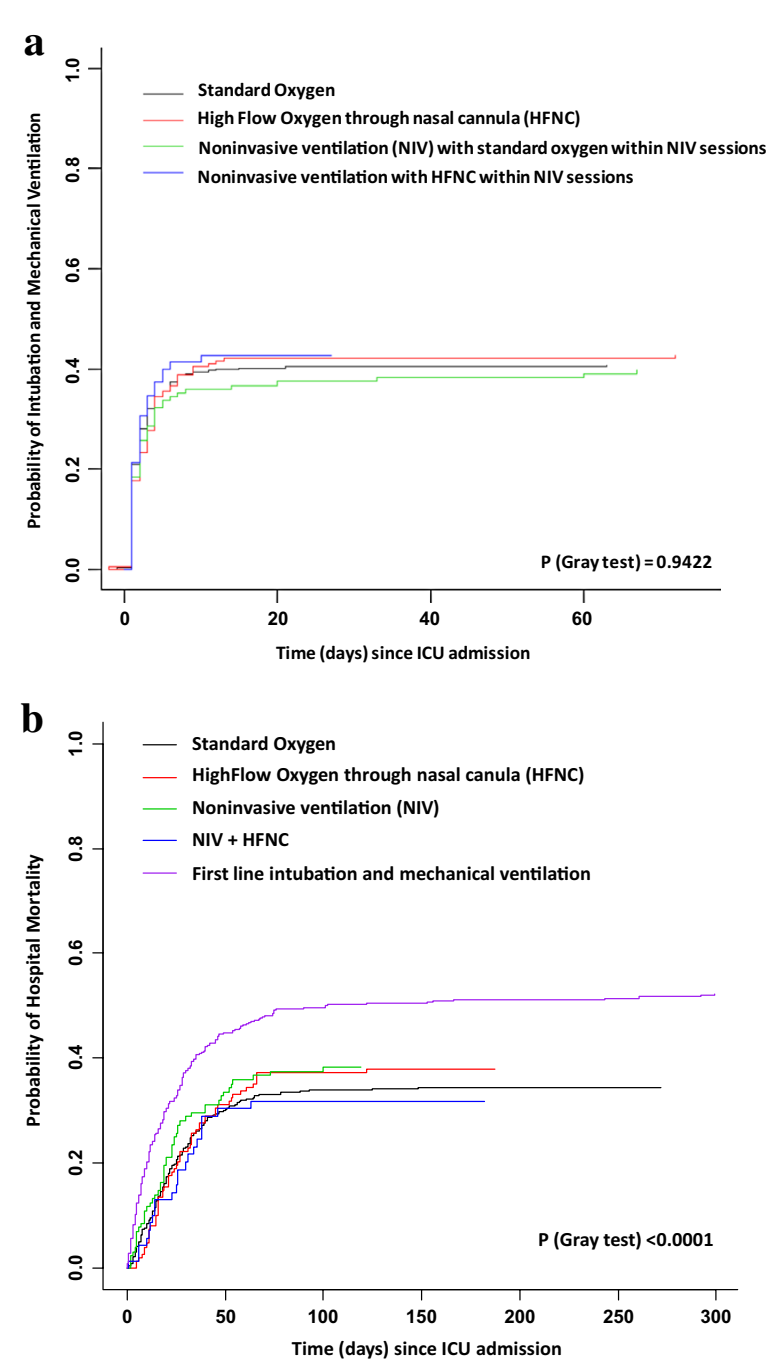

Fig. 2 a Cumulative incidence of intubation according to the ventilation mode on ICU admission. This is restricted to the 915 patients not intubated on ICU admission. b Cumulative incidence of hospital mortality according to the ventilation mode on ICU admission. This includes all the 1545 patients for whom status at hospital discharge was available
$80 \%$ (60-100), $70 \%(50-100)$, and 50\% (40-75) at day 1 , day 2 , and day 3 , respectively. Among patients who were intubated, initial settings were tidal volume of $6.7 \mathrm{~mL} / \mathrm{kg}$ (6-7.5) ideal body weight and PEEP of $8 \mathrm{cmH}_{2} \mathrm{O}(6-12)$.

Cumulative incidence of intubation and hospital mortality are reported in Fig. 2a, b. By multivariable analysis (Fig. 3), factors associated with the cause-specific hazard of intubation and ventilation were age (hazard ratio $=0.92 /$ year, $95 \%$ CI $0.86-0.99)$, day- 1 SOFA score (1.09/point, 1.06-1.13), $\mathrm{PaO}_{2} / \mathrm{FiO}_{2}<300$ at day 1 (1.47, 1.05-2.07), and ARF etiology [Pneumocystis jirovecii pneumonia, 2.11 (1.42-3.14); invasive pulmonary aspergillosis, 1.85 (1.21-2.85); and undetermined etiology, $1.46(1.09-1.98)]$. The cause-specific hazard of intubation and ventilation associated with HFNC use was 0.77 (0.59-1.01), $P=0.06$. After matching on propensity score of being administered HFNC (Supplemental Fig. 2), there was an effect from HFNC on the cause-specific hazard of intubation $(\mathrm{HR}=0.77,95 \% \mathrm{CI} 0.59-1.00, P=0.05)$. After matching on propensity score of being administered NIV (Supplemental Fig. 2), there was no effect of NIV on the cause-specific hazard of intubation (HR $=0.90,95 \% \mathrm{CI}$ $0.67-1.21, P=0.48$ ).

Tables 2 and 3 compare patients' characteristics according to status at hospital discharge. By multivariable analysis (Fig. 4), determinants of hospital mortality were age [odds ratio/year $=1.18(1.09-1.27)$ ], direct admission to the ICU [0.69 (0.54-0.87)], day-1 SOFA without respiratory score [1.12/point (1.08-1.16)], $\mathrm{PaO}_{2} / \mathrm{FiO}_{2}<100$ [1.60 (1.03-2.48)], and undetermined ARF etiology [1.43 (1.04-1.97)]. The need for intubation was also associated with hospital mortality; however, the magnitude of the effect depended on the timing of intubation and initial oxygenation strategy. For instance, odds for mortality were 2.55 (95\% confidence interval, 1.94-3.29) after first-line IMV, 2.31 (1.09-4.91) after failure of HFNC + NIV, 3.65 (2.05-6.53) after failure of NIV alone, 4.16 (2.91-5.93) after failure of standard oxygen, and 5.54 (3.27-9.38) after failure of HFNC alone. On the basis of matched samples from the eligible population of patients with available mode of oxygenation or ventilation at ICU admission and status at hospital discharge, there was no evidence of any survival benefit from NIV $(\mathrm{OR}=0.98,95 \%$ CI 0.9-0.1.1, $P=0.69)$, nor from HFNC $(\mathrm{OR}=1.0,95 \%$ CI $0.9-1.1, P=0.86)$. When restricting these analyses to the population of 859 patients not intubated at ICU admission and free of any DNI order, and after excluding patients with no available status at hospital discharge, results were not markedly modified, with still no survival benefit from NIV $(\mathrm{OR}=1.0,95 \%$ CI $0.9-1.1, P=0.68)$ or from HFNC $(\mathrm{OR}=1.0,95 \% \mathrm{CI}$ $0.9-1.1, P=0.57)$. 
Hazard Ratios

(95\% Confidence Intervals)

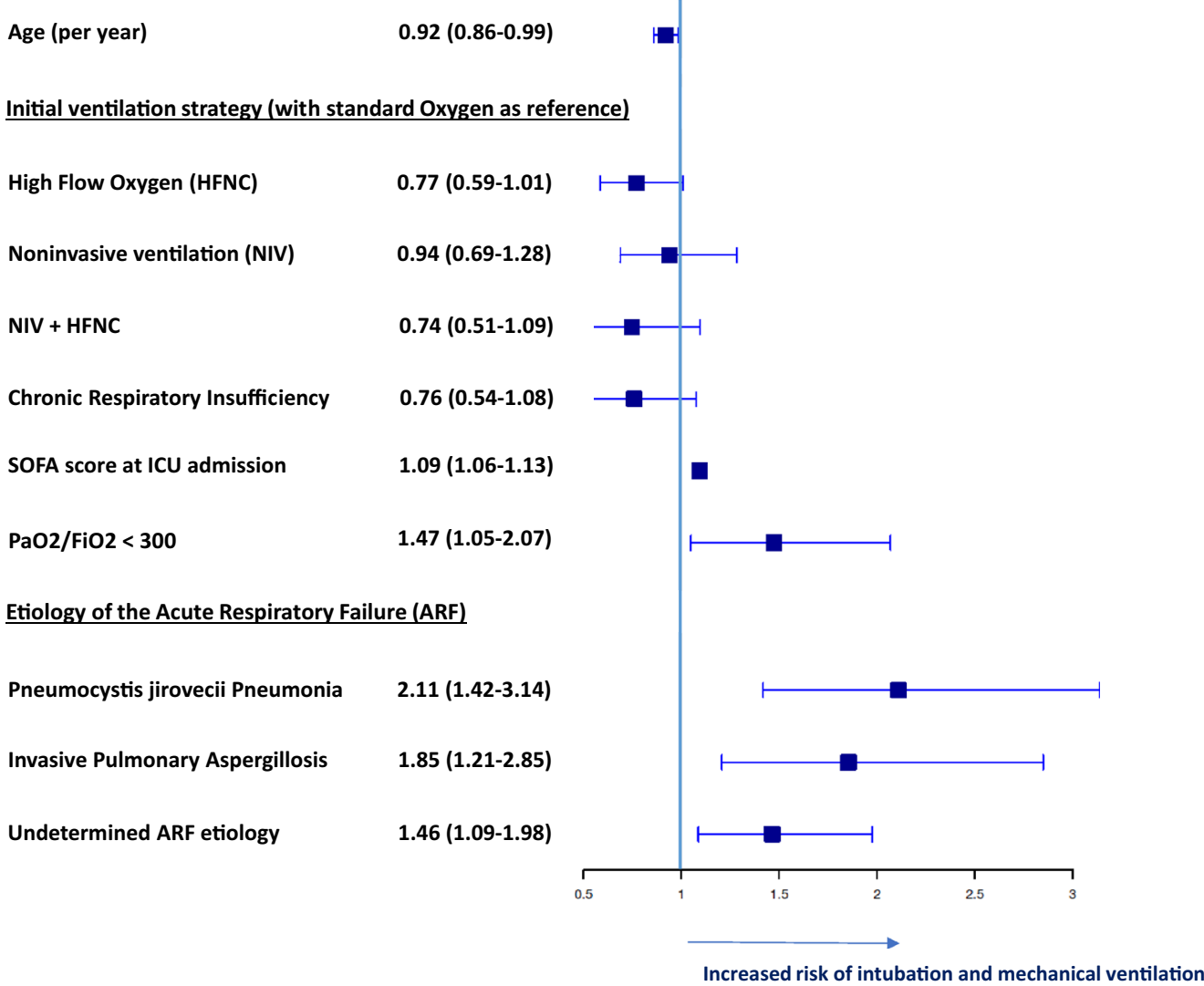

Fig. 3 Multivariate model of the cause-specific hazard of intubation. This analysis is restricted to the 915 patients not intubated on ICU admission. Plots report variables independently associated with the need for intubation in the final model, with their $95 \%$ confidence intervals

Sensitivity analyses performed after multiple imputation of missing data, or in the subgroup of patients with hematological malignancies, or restricted to the group of patients with full code status led to the same results. When the center was introduced as a cluster effect in the regression models, it was not significantly associated with outcomes (hospital mortality, $P=0.30$; intubation, $P=0.76$, Supplemental Fig. 3).

Compared to patients with established causes of ARF, those with undetermined ARF etiology had longer symptoms prior to ICU admission [ 1 day $(0-4)$ vs. 2 days $(0-5), P=0.008$ ], were more frequently receiving antifungal prophylaxis $(50.3 \%$ vs. $41.8 \%, P=0.04)$, had a higher SOFA score excluding respiratory score on day 1 [5 (3-8) vs. $4(2-8), P=0.03$ ], were more frequently intubated on day 1 or $2(56.4 \%$ vs. $47.9 \%, P=0.05)$, and had higher hospital mortality rate $(52.8 \%$ vs. $42.8 \%$, $P=0.009)$. Interestingly, the number of FO-BAL performed were not different (62\% vs. $59 \%, P=0.79$ ). Also, the type and number of noninvasive diagnostic tests including sputum samples, serum biomarkers, and computed tomography scan were not significantly different between the two groups.

\section{Discussion}

We report a large, prospective multicenter multinational cohort study on critically ill immunocompromised patients with ARF. HFNC has an effect on intubation rate but did not influence all-cause hospital mortality. Use of NIV did not impact outcomes. The need for intubation was associated with mortality with higher odds for mortality in case of NIV or HFNC failure. Most importantly, the cause of ARF influenced IMV and mortality rates, particularly when ARF etiology remained unknown despite comprehensive diagnostic workup, suggesting that any effort towards a diagnosis may trump avoiding intubation.

The hospital mortality of $44.1 \%$ stresses the need to maintain vigilance to improve outcomes in this group of patients that is growing and will develop more complications in the future [26]. Of those patients who were not intubated on admission, 39\% needed IMV, which is consistent with recent studies [11, 14]. 
Table 2 Patient's characteristics according to status at hospital discharge

\begin{tabular}{|c|c|c|c|}
\hline Numbers (\%) or median (IQR) & Patients discharged alive, $N=863$ & Patients died during hospitalization, $N=682$ & $P$ value \\
\hline Age (years) & $63.0(52.8-70.6)$ & $64.8(56.6-72.8)$ & 0.001 \\
\hline Female gender & $346(40.1 \%)$ & $267(39.1 \%)$ & 0.78 \\
\hline Chronic pulmonary disease & $62(16.9 \%)$ & $23(3.4 \%)$ & 0.30 \\
\hline Chronic cardiac failure & $107(20.0 \%)$ & $31(4.5 \%)$ & 0.005 \\
\hline Poor performance status (ECOG > 2) & $311(36.0 \%)$ & $364(53.4)$ & $<0.0001$ \\
\hline Symptoms duration prior ICU & $1(0-4)$ & $1(0-4)$ & 0.96 \\
\hline Location before ICU admission & & & 0.08 \\
\hline Emergency department & $223(25.8 \%)$ & $142(20.8 \%)$ & \\
\hline Hospital wards & $467(54.1 \%)$ & $417(61.1 \%)$ & \\
\hline Other ICUs & $56(6.5 \%)$ & $48(7.0 \%)$ & \\
\hline Other locations & $117(13.6 \%)$ & $75(11 \%)$ & \\
\hline Direct admission to the ICU & $324(37.6 \%)$ & $188(27.6 \%)$ & $<0.0001$ \\
\hline Nightly/weekend admissions & $524(60.7 \%)$ & $393(57.6 \%)$ & 0.24 \\
\hline \multicolumn{4}{|l|}{ Underlying conditions } \\
\hline Hematological malignancy & $438(50.75 \%)$ & $362(53.2 \%)$ & 0.39 \\
\hline Solid tumor & $295(34.2 \%)$ & $249(36.5 \%)$ & 0.37 \\
\hline Systemic diseases & $163(18.9 \%)$ & $106(15.5 \%)$ & 0.09 \\
\hline Solid organ transplant & $97(11.2 \%)$ & $59(8.6 \%)$ & 0.13 \\
\hline Drug-related immunosuppression & $10(1.2 \%)$ & $3(0.4 \%)$ & 0.21 \\
\hline Neutropenia & $122(14.1 \%)$ & $130(19.1 \%)$ & 0.01 \\
\hline Hematopoietic stem cell transplantation & & & 0.16 \\
\hline Autologous & $50(5.8 \%)$ & $45(6.6 \%)$ & \\
\hline Allogeneic & $71(8.2 \%)$ & $74(10.9 \%)$ & \\
\hline Days since diagnosis & $156(18-734)$ & $120(11-578)$ & 0.26 \\
\hline Newly diagnosed disease & $241(27.9 \%)$ & $216(31.7 \%)$ & $<0.0001$ \\
\hline Day-1 SOFA score & $6(3-9)$ & $8(5-11)$ & $<0.0001$ \\
\hline Day-1 SOFA without respiratory items & $4(1-7)$ & $6(3-9)$ & $<0.0001$ \\
\hline $\mathrm{PaO}_{2} / \mathrm{FiO}_{2}$ on day 1 & $173(114-210)$ & $158(100-195)$ & $<0.0001$ \\
\hline $\mathrm{PaO}_{2} / \mathrm{FiO}_{2}$ classes & & & 0.02 \\
\hline$\geq 300 / 200-299$ & $91(10.5 \%) / 156(18.1 \%)$ & $52(7.6 \%) / 159(23.3 \%)$ & \\
\hline $100-199 /<100$ & $469(54.3 \%) / 147(17.0 \%)$ & $363(53.2 \%) / 108(15.8 \%)$ & \\
\hline Goals of care on ICU admission & & & $<0.0001$ \\
\hline Full code status & $802(92.9 \%)$ & $566(83.0 \%)$ & \\
\hline Time-limited trial & $13(1.5 \%)$ & $51(7.5 \%)$ & \\
\hline DNR/DNI & $20 / 28(5.6 \%)$ & $30 / 35(9.5 \%)$ & \\
\hline Need for intubation and ventilation & & & $<0.0001$ \\
\hline No & $461(53.4 \%)$ & $163(23.9 \%)$ & \\
\hline Within $2 \mathrm{~h}$ of ICU admission & $261(30.2 \%)$ & $261(30.2 \%)$ & \\
\hline After failure of standard oxygen & $75(8.7 \%)$ & $112(16.4 \%)$ & \\
\hline After noninvasive ventilation (NIV) failure & $24(2.8 \%)$ & $30(4.4 \%)$ & \\
\hline After high-flow oxygen (HFNC) failure & $26(3.0 \%)$ & $50(7.3 \%)$ & \\
\hline After failure of NIV + HFNC & $16(1.8 \%)$ & $14(2.0 \%)$ & \\
\hline
\end{tabular}

The analysis did not include 66 patients in whom hospital mortality was missing

NIV use in critically ill immunocompromised patients with ARF was believed to be the gold standard at a time where mortality associated with standard oxygen was above $80 \%$, but only $50 \%$ with NIV [27]. A post hoc analysis of a HFNC trial reported that NIV was independently associated with increased mortality [11]. However, this was not confirmed in a randomized trial that found no benefit and no harm from NIV [14]. Similarly, in the present study, NIV did not influence the need for IMV or mortality rates: among the 237 patients receiving NIV, 
Table 3 Association between acute respiratory failure etiologies and hospital mortality

\begin{tabular}{|c|c|c|c|}
\hline Numbers (\%) or median (IQR) & $\begin{array}{l}\text { Patients discharged alive from the hospi- } \\
\text { tal, } N=863(55.8 \%)\end{array}$ & $\begin{array}{l}\text { Patients who died before hospital dis- } \\
\text { charge, } N=682(44.2 \%)\end{array}$ & $P$ value \\
\hline Bacterial infection & & & 0.27 \\
\hline Clinically documented & $125(14.5 \%)$ & $84(12.3 \%)$ & \\
\hline Microbiologically documented & $129(14.9 \%)$ & $118(17.3 \%)$ & \\
\hline \multicolumn{4}{|l|}{ Viral infection } \\
\hline Influenza & $86(10.0 \%)$ & $66(9.7 \%)$ & 0.92 \\
\hline Other viruses & $138(16.0 \%)$ & $106(15.5 \%)$ & 0.87 \\
\hline Septic shock from extrathoracic source & $81(9.4 \%)$ & $68(10.0 \%)$ & 0.76 \\
\hline \multicolumn{4}{|l|}{ Invasive fungal infection (IFI) } \\
\hline $\begin{array}{l}\text { Proven or probable invasive pulmonary } \\
\text { aspergillosis (IPA) }\end{array}$ & $30(3.5 \%)$ & $31(4.5 \%)$ & 0.35 \\
\hline Pneumocystis jirovecii pneumonia & $34(3.9 \%)$ & $35(5.1 \%)$ & 0.32 \\
\hline Candidemia with septic shock & $15(1.7)$ & $21(3.1 \%)$ & 0.12 \\
\hline $\begin{array}{l}\text { All IFI cases including other fungi and pos- } \\
\text { sible cases of IPA }\end{array}$ & $105(12.2 \%)$ & $121(17.7 \%)$ & 0.003 \\
\hline Aspiration pneumonia & $38(4.4 \%)$ & $31(4.5 \%)$ & 0.99 \\
\hline Airway-related disorders & $38(4.4 \%)$ & $16(2.3 \%)$ & 0.04 \\
\hline Drug-related pulmonary toxicity & $34(3.9 \%)$ & $19(2.8 \%)$ & 0.27 \\
\hline Disease-related infiltrates & $69(8.0 \%)$ & $73(10.7 \%)$ & 0.08 \\
\hline Cardiogenic pulmonary edema & $66(7.6 \%)$ & $33(4.8 \%)$ & 0.03 \\
\hline Undetermined & 94 (10.9\%) & $105(15.4 \%)$ & 0.01 \\
\hline More than one ARF etiology & $125(14.5 \%)$ & 105 (15.4\%) & 0.67 \\
\hline
\end{tabular}

90 (38\%) subsequently required IMV, so did patients receiving standard oxygen or HFNC. The same applied to hospital mortality. Regarding HFNC, a post hoc analysis restricted to immunocompromised patients with ARF reported that ORs for intubation were higher in patients treated with NIV than in those treated with HFNC [11]. However, this was not confirmed by the post hoc analysis of the NIV trial in which HFNC was neither associated with a lower intubation rate nor day-28 mortality [14]. In the present study, HFNC, which was used in more severely hypoxemic patients, has an effect of IMV rate. Last, the association of HFNC + NIV was not associated with increased intubation or hospital mortality.

Another major finding from this study is the association between the cause of ARF and outcomes. More particularly, patients with ARF from unknown cause in spite of extensive workup were at high risk of IMV and death. The prevalence of $13.2 \%$ of unknown diagnoses is in agreement with previous reports [18, 23, 27, 28]. Also, this study confirms that these patients underwent as much diagnostic efforts as patients with identified ARF etiology. That patients with undetermined cause of ARF have poor outcomes could be the result of delay and failure to recognize and treat a pulmonary complication. However, more research is needed to understand whether this clinical entity does actually refer to unidentified pathogens, unknown toxicity, or atypical organ involvement by the disease. For now, this result suggests that more data should be gathered on ARF from undetermined etiology to guide clinicians towards a more aggressive diagnostic strategy, including cytokine concentrations, as well as molecular diagnostics in blood and BAL, and minimally invasive CT-guided lung biopsies, cryobiopsies, or surgical biopsies. Meanwhile, empirical steroids, antifungals, or antiviral agents may also be considered despite the lack of evidence. Furthermore, this study suggests that in addition to adjusting oxygenation strategy to a patient's severity, clinicians should also focus on how the selected oxygenation device enables an appropriate diagnostic workup.

Strengths of our study include the multicenter and multinational design and a large number of patients, making this study the largest to date. Also, follow-up and hospital mortality were missing in only $4 \%$ of the patients. The profile of infectious diseases in our population indicates severe immunologic impairment. These results also have a high degree of external validity, since the centers belong to a large multinational study group including university and nonuniversity hospitals. We found no evidence that oxygenation modalities influenced any of the endpoints or were beneficial in some subgroups. Moreover, NIV and HFNC were administered optimally in terms of duration and settings. Overall, now that NIV use is either neutral [14] 


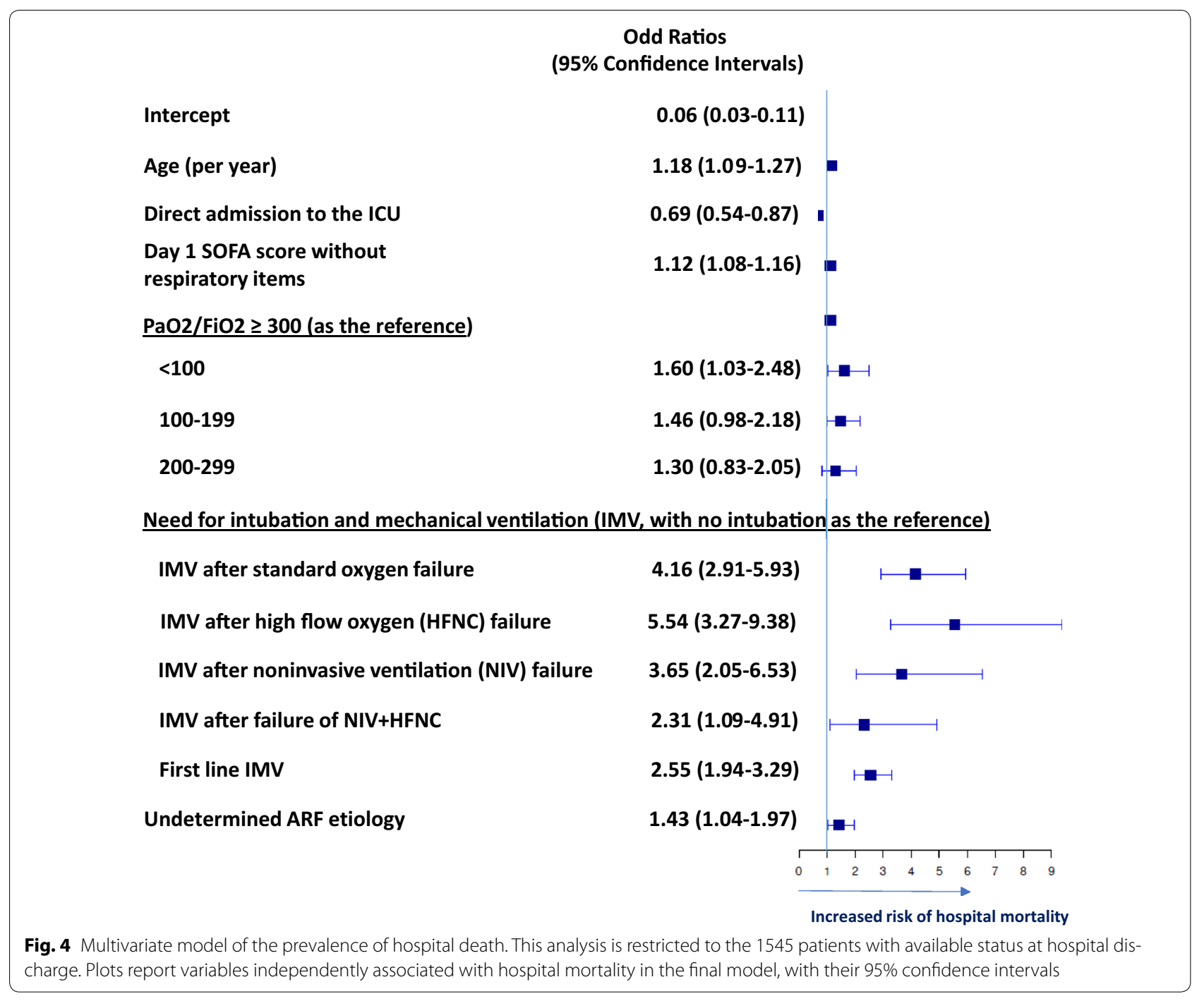

or associated with increased mortality [11], NIV can hardly be part of the standard of care in this setting until trials adequately powered and with appropriate control groups demonstrate that a better NIV administration (continuous and prolonged NIV or helmet-based continuous positive airway pressure) translates into improved survival. Also, in immunocompromised patients with hypoxemic ARF requiring intubation, no trial has assessed benefits from NIV or HFNC as demonstrated in non-immunocompromised patients [29]. Regarding HFNC, no impact was found regarding mortality. Specific trials of oxygen therapy in immunocompromised patients with hypoxemic ARF are needed before any conclusion can be drawn.

The present study has several limitations. First, the observational design precludes strong conclusions; however, the use of propensity score matching, which allowed us to erase main imbalances in potential confounders across oxygenation groups, reached conclusions that were similar to those from regression modelling. Second, criteria for intubation, management decisions, or diagnostic and therapeutic strategies were not standardized across centers. However, use of NIV, HFNC, and IMV rates were not significantly different across centers, and no center effect was found. Similarly, the number of diagnostic procedures and the proportion of patients left with undetermined ARF etiologies were not significantly different across centers; nevertheless, such a multicenter design argues for the good external validity of our results. Third, debates about ventilation and oxygenation modalities often arise in studies reporting no benefit. However, the 8-h median NIV duration per day and HFNC settings observed in the present study are consistent with those in other recent reports [11-13]. Studies comparing HFNC use versus standard oxygen are needed. Last, earlier and more 
aggressive diagnostic and therapeutic strategies should be developed and validated for patients with ARF from undetermined etiology. Lung biopsies are difficult to perform in patients with refractory hypoxemia and frequent hemostatic disorders and thrombocytopenia. Also, the value of rescue ECMO to increase time to treatment response or to support lung biopsy is unclear, as reported outcomes in immunocompromised patients are inconsistent, however, grim in specific subgroups [30].

\section{Conclusions}

Among immunocompromised patients admitted to ICU with hypoxemic acute respiratory failure, initial oxygenation strategy has an impact on IMV rate but not on mortality. However, patients with ARF from unknown etiology are more likely to be intubated and have a higher hospital mortality. Clinicians managing these patients should increase their ability to identify and treat the cause of pulmonary involvement.

\section{Electronic supplementary material}

The online version of this article (doi:10.1007/s00134-017-4947-1) contains supplementary material, which is available to authorized users.

\section{Author details}

${ }^{1}$ Medical Intensive Care Unit, APHP, Hôpital Saint-Louis, Famirea Study Group, ECSTRA team, and Clinical Epidemiology, UMR 1153, Center of Epidemiology and Biostatistics, Sorbonne Paris Cité, CRESS, INSERM, Paris Diderot Sorbonne University, Paris, France. ${ }^{2}$ The Department of Intensive Care Medicine (710), Radboud University Medical Center, Nijmegen, The Netherlands. ${ }^{3}$ The Department of Critical Care and Graduate Program in Translational Medicine, D'Or Institute for Research and Education, Programa de Pós-Graduação em Clínica Médica, Rio De Janeiro, Brazil. ${ }^{4}$ Department of Intensive Care, Rigshospitalet, University of Copenhagen, Copenhagen, Denmark. ${ }^{5}$ CIBERES, Universitat Autonòma de Barcelona, European Study Group of Infections in Critically III Patients (ESGCIP), Barcelona, Spain. ${ }^{6}$ Pulmonary and Critical Care Medicine, Mayo Clinic, Rochester, MN, USA. ${ }^{7}$ Division of Pulmonary and Critical Care, Penn State University College of Medicine, Hershey, PA, USA. ${ }^{8}$ Department of Intensive Care, Hôpital Erasme, Université Libre de Bruxelles (ULB), Brussels, Belgium. ${ }^{9}$ Department of Intensive Care Medicine, Multidisciplinary Intensive Care Research Organization (MICRO), St. James's Hospital, Dublin, Ireland. ${ }^{10}$ Department of Clinical Medicine, Trinity College, Wellcome Trust-HRB Clinical Research Facility, St James Hospital, Dublin, Ireland. ${ }^{11}$ Department of Medicine I, Medical University of Vienna, Vienna, Austria. ${ }^{12}$ Department of Anesthesiology and Intensive Care Medicine and Institute for Medical Humanities, 1st Faculty of Medicine, Charles University in Prague and General University Hospital, Prague, Czech Republic. ${ }^{13} \mathrm{CHU}$ Grenoble Alpes, Service de réanimation médicale, Faculté de Médecine de Grenoble, INSERM, U1042, Université Grenoble-Alpes, Grenoble, France. ${ }^{14}$ Department of Medicine and Interdepartmental Division of Critical Care Medicine, Sinai Health System, University of Toronto, Toronto, ON, Canada. ${ }^{15}$ Agostino Gemelli University Hospital, Università Cattolica del Sacro Cuore, Rome, Italy. ${ }^{16}$ Department of Medical Intensive Care Medicine, University Hospital of Angers, Angers, France. ${ }^{17}$ Department of Emergencies and Critical Care, Oslo University Hospital, Oslo, Norway. ${ }^{18}$ Division of Intensive Care Medicine, Department of Anesthesiology, Intensive Care and Pain Medicine, University of Helsinki and Helsinki University Hospital, Helsinki, Finland. ${ }^{19}$ Department of Critical Care, University Medical Center Groningen, Groningen, The Netherlands. ${ }^{20}$ Center Hospitalier de Versailles, Medical-Surgical Intensive Care Unit, Le Chesnay, France. ${ }^{21}$ Department of Anesthesiology I, Herlev University Hospital, Herlev, Denmark. ${ }^{22}$ Medical ICU, Cochin Hospital, Assistance Publique-Hôpitaux de Paris and University
Paris Descartes, Paris, France. ${ }^{23}$ King's College Hospital, London SE5 9RS, UK. ${ }^{24}$ Critical Care Center, CHU Lille, School of Medicine, University of Lille, Lille, France. ${ }^{25}$ Terapia Intensiva, Hospital Maciel, Montevideo, Uruguay. ${ }^{26}$ Normandie Univ, UNIROUEN, EA-3830, Department of Medical Intensive Care, Rouen University Hospital, 76000 Rouen, France. ${ }^{27}$ ICU, Fundação Pio XII, Hospital de Câncer de Barretos, Barretos, Brazil. ${ }^{28}$ Medical Intensive Care Unit, La Source Hospital, CHR Orléans, Orléans, France. ${ }^{29}$ Intensive Care Department, University of Southern Denmark, Odense, Denmark. ${ }^{30}$ Department of Anaesthesia and Intensive Care, Odense University Hospital, Odense, Denmark. ${ }^{31}$ Medical Intensive Care Unit, Hôtel Dieu-HMEUniversity Hospital of Nantes, Nantes, France. ${ }^{32}$ Réanimation Polyvalente et Département d'Anesthésie et de Réanimation, Institut Paoli-Calmettes, Marseille, France. ${ }^{33}$ ECSTRA Team, Biostatistics and Clinical Epidemiology, UMR 1153, INSERM, Paris Diderot Sorbonne University and Service de Biostatistique et Information Médicale AP-HP, Hôpital Saint-Louis, Paris, France.

\section{Acknowledgements}

This study was performed on behalf of the "Caring for critically ill immunocompromised patients-Multinational Network (Nine-I). This group includes critical care specialists from 16 countries in Europe, the USA, Canada and South America. The primary aim of this group is to improve and standardize practices in the management of critically ill immunocompromised patients.

Contributors: Antoine Rabbat, Hôpital Cochin, Paris, France; Isabelle Vinatier, CHD de Vendée, La Roche Sur Yon, France; Michael Darmon, CHU, SaintEtienne, France; Kada Klouche, Laura Platon, CHU, Montpellier, France; Martine Nyunga, CHG Victor Provo, Roubaix, France; Alexandre Demoule, Julien Mayaux, CHU Pitié-Salpétrière, Paris, France; Florent Wallet, CHU, Lyon Sud, France; Akli Chermak, CH Sud Essonne, Etampes, France; Amelie Seguin Réanimation Medicale - CHU de Caen, Caen, France; Caroline Lemaitre, Elise Artaud-Macari, University Hospital, Medical Intensive Care, Rouen, France; Jonas Nelsen, Rigshospitalet, Copenhagen, Denmark; Ann M. Moeller, Herlev University Hospital, UCPH, Herlev, Denmark; Thomas Kaufmann, Department of Critical Care, Groningen, the Netherlands; Dennis Bergmans, Department of Critical Care, Maastricht, the Netherlands; Angélique Spoelstra de Man, Department of Critical Care, Amsterdam, the Netherlands; Ana Paula Pierre de Moraes, Hospital de Câncer do Maranhao, Brazil; William Viana, Hospital Copa d'Or, Brazil; Guilliana Moralez, Hospital GetulioVargas, Rio de Janeiro, Brazil; Thiago Lishoa, Hospital Santa Rita, Santa Casa de Misericordia, Porte Allegre, Brazil; Thiago Domingos Correa, ICU, Hospital Israelita Albert Einstein, São Paulo, Brazil; Belen Encina, Val Hebron, Barcelona, Spain; Gabriel Moreno, Department of Critical Care, Bellitge, Spain; Emilio Rodriguez Luis, Department of Critical Care, Santiago de compostella, Spain; Llorenç Socias Crespi, Department of Critical Care, Palma, Spain; Yadav Hemang, Pulmonary and Critical Care Medicine, Mayo Clinic, Rochester, MN, USA; Anne-Pascale Meert, Institut Jules Bordet, Brussels, Belgique; Dominique Benoit, Ghent University Hospital, Ghent, Belgique; Nina Buchtele, Department of Medicine I, Vienna, Austria; Thomas Staudinger, Department of Medicine I, Vienna, Austria; Gottfried Heinz, Department of Medicine II, Vienna, Austria; Gürkan Sengölge, Department of Medicine III, Vienna, Austria; Christian Zauner, Department of Medicine III, Vienna, Austria; Peter Jaksch, Department of Thoracic Surgery, Vienna, Austria; Karin Amrein, Department of Internal Medicine, Graz, Austria; Aisling Mc Mahon, Department of Critical Care, St James, Dublin, Ireland; Brian Marsh, Department of Critical Care, Mater misericordia, Dublin, Ireland; Balik Martin, Department of Critical Care, Prague, Czech Republic; Karvunidis Thomas, Department of Critical Care, Pielsa, Czech Republic; Pål Klepstad, St. Olavs Hospital, Trondheim, Norway; Anne Kuitunen, Department of Critical Care, Tempere, Finland; Gilda Cinnella, Antonella Cotoia, Ospedali Riuniti, Department of Critical Care, Foggia, Italy; Sumech Shah, Mount Sinai Hospital, Canada.

\section{Compliance with ethical standards}

\section{Conflicts of interest}

The authors declare no conflict of interest in relation to this study.

Received: 9 September 2017 Accepted: 18 September 2017

Published online: 25 September 2017 


\section{References}

1. Harpaz R, Dahl RM, Dooling KL (2016) Prevalence of immunosuppression among US adults, 2013. JAMA 316(23):2547-2548

2. Fernandez-Ruiz M, Kumar D, Humar A (2014) Clinical immune-monitoring strategies for predicting infection risk in solid organ transplantation. Clin Transl Immunol 3(2):e12

3. Winthrop KL, Novosad SA, Baddley JW et al (2015) Opportunistic infections and biologic therapies in immune-mediated inflammatory diseases: consensus recommendations for infection reporting during clinical trials and postmarketing surveillance. Ann Rheum Dis 74(12):2107-2116

4. Azoulay E, Pene F, Darmon M et al (2015) Managing critically ill hematology patients: time to think differently. Blood Rev 29(6):359-367

5. Puxty K, McLoone P, Quasim T, Sloan B, Kinsella J, Morrison DS (2015) Risk of critical illness among patients with solid cancers: a population-based observational study. JAMA Oncol 1(8):1078-1085

6. Siegel RL, Miller KD, Jemal A (2017) Cancer statistics, 2017. CA Cancer J Clin 67(1):7-30

7. Morrison VA (2014) Immunosuppression associated with novel chemotherapy agents and monoclonal antibodies. Clin Infect Dis 59(Suppl 5):S360-\$364

8. Novosad SA, Winthrop KL (2014) Beyond tumor necrosis factor inhibition: the expanding pipeline of biologic therapies for inflammatory diseases and their associated infectious sequelae. Clin Infect Dis 58(11):1587-1598

9. Canet E, Zafrani L, Azoulay E (2016) The critically ill kidney transplant recipient: a narrative review. Chest 149(6):1546-1555

10. Dumas G, Geri G, Montlahuc C et al (2015) Outcomes in critically ill patients with systemic rheumatic disease: a multicenter study. Chest 148(4):927-935

11. Frat JP, Ragot S, Girault C et al (2016) Effect of non-invasive oxygenation strategies in immunocompromised patients with severe acute respiratory failure: a post hoc analysis of a randomised trial. Lancet Respir Med 4(8):646-652

12. Lemiale $V$, Resche-Rigon M, Mokart D et al (2017) High-flow nasal cannula oxygenation in immunocompromised patients with acute hypoxemic respiratory failure: a Groupe de Recherche Respiratoire en Reanimation Onco-Hematologique Study. Crit Care Med 45(3):e274-e280

13. Mokart D, Geay C, Chow-Chine L et al (2016) High-flow oxygen therapy in cancer patients with acute respiratory failure. Intensive Care Med 41(11):2008-2010

14. Lemiale V, Mokart D, Resche-Rigon M et al (2015) Effect of noninvasive ventilation vs oxygen therapy on mortality among immunocompromised patients with acute respiratory failure: a randomized clinical trial. JAMA 314(16):1711-1719

15. Papazian L, Corley A, Hess D et al (2016) Use of high-flow nasal cannula oxygenation in ICU adults: a narrative review. Intensive Care Med 42(9):1336-1349

16. Mokart D, Lambert J, Schnell D et al (2013) Delayed intensive care unit admission is associated with increased mortality in patients with cancer with acute respiratory failure. Leuk Lymphoma 54(8):1724-1729
17. Burghi G, Lemiale V, Seguin A et al (2011) Outcomes of mechanically ventilated hematology patients with invasive pulmonary aspergillosis. Intensive Care Med 37(10):1605-1612

18. Contejean A, Lemiale V, Resche-Rigon M et al (2017) Increased mortality in hematological malignancy patients with acute respiratory failure from undetermined etiology: a Groupe de Recherche en Reanimation Respiratoire en Onco-Hematologique (Grrr-OH) study. Ann Intensive Care 6(1):102

19. Azoulay E, Mokart D, Pene F et al (2013) Outcomes of critically ill patients with hematologic malignancies: prospective multicenter data from France and Belgium - a groupe de recherche respiratoire en reanimation onco-hematologique study. J Clin Oncol 31(22):2810-2818

20. Azoulay E, Lemiale V, Mokart D et al (2014) Acute respiratory distress syndrome in patients with malignancies. Intensive Care Med 40(8):1106-1114

21. Adda M, Coquet I, Darmon M, Thiery G, Schlemmer B, Azoulay E (2008) Predictors of noninvasive ventilation failure in patients with hematologic malignancy and acute respiratory failure. Crit Care Med 36(10):2766-2772

22. Gristina GR, Antonelli M, Conti G et al (2011) Noninvasive versus invasive ventilation for acute respiratory failure in patients with hematologic malignancies: a 5-year multicenter observational survey. Crit Care Med 39(10):2232-2239

23. Azoulay E, Mokart D, Lambert J et al (2010) Diagnostic strategy for hematology and oncology patients with acute respiratory failure: randomized controlled trial. Am J Respir Crit Care Med 182(8):1038-1046

24. Vincent JL, Moreno R, Takala J et al (1996) The SOFA (Sepsis-related organ failure assessment) score to describe organ dysfunction/failure. On behalf of the Working Group on Sepsis-Related Problems of the European Society of Intensive Care Medicine. Intensive Care Med 22(7):707-710

25. Del Sorbo L, Ranieri VM, Ferguson ND (2016) The Berlin definition met our needs: yes. Intensive Care Med 42(5):643-647

26. Amos SM, Duong CP, Westwood JA et al (2011) Autoimmunity associated with immunotherapy of cancer. Blood 118(3):499-509

27. Hilbert G, Gruson D, Vargas F et al (2001) Noninvasive ventilation in immunosuppressed patients with pulmonary infiltrates, fever, and acute respiratory failure. N Engl J Med 344(7):481-487

28. Rano A, Agusti C, Benito N et al (2002) Prognostic factors of non-HIV immunocompromised patients with pulmonary infiltrates. Chest 122(1):253-261

29. Antonelli M, Conti G, Rocco M et al (1998) A comparison of noninvasive positive-pressure ventilation and conventional mechanical ventilation in patients with acute respiratory failure. N Engl J Med 339(7):429-435

30. Wohlfarth P, Ullrich R, Staudinger T et al (2014) Extracorporeal membrane oxygenation in adult patients with hematologic malignancies and severe acute respiratory failure. Crit Care 18(1):R20 